UCI-TR-2007-31

\title{
Determining Spins of Metastable Sleptons at the Large Hadron Collider
}

\author{
Arvind Rajaraman and Bryan T. Smith \\ Department of Physics and Astronomy, \\ University of California, Irvine, CA 92697, USA
}

\begin{abstract}
We investigate models in minimal supergravity parameter space which contain metastable sleptons. We find the luminosity required to determine the slepton spin in these scenarios, and apply our analysis to two benchmark models. We show that the spin of the slepton in one of the benchmark models can be determined with less than $30 \mathrm{fb}^{-1}$ of data, while the slepton spin in the other model can be determined with roughly $40 \mathrm{fb}^{-1}$ of data. We show how our analysis can be applied to other models, and give an estimate of the luminosity needed for a spin determination as a function of slepton mass. This analysis can be used to distinguish supersymmetry and extra dimensions.
\end{abstract}

PACS numbers: 04.65.+e, 12.60.Jv, 13.85.-t 


\section{INTRODUCTION}

In the near future, the LHC will begin taking data, and there are high expectations that there will be a discovery of new physics beyond the Standard Model. It will be a further challenge to isolate the nature of the new physics from the many possible scenarios. In particular, supersymmetric models can appear very similar to models of extra dimensions, and it is often difficult to tell them apart [1]. In fact, the best way to distinguish these two classes of models is by measuring the spins of the new particles.

Motivated by an effort to distinguish between supersymmetry and extra dimensions, there has been a great deal of work done on measuring the spins of beyond the standard model (BSM) particles. These studies have mostly focused on the case of missing energy signals [2, 3, 4, 5, 6, 7, 8, 9]. These works considered cascades of heavier particles decaying down to a neutral LSP, looking at the kinematic distribution of jets and leptons.

In this note we shall discuss a different class of models, in which the signals do not come from missing energy, but instead from metastable charged particles. We shall focus on the possibility of measuring the spins of these new particles, and thereby distinguishing supersymmetry from extra dimensions.

The models we discuss are part of minimal supergravity (mSugra) [10] parameter space. In these models, the gravitino is the lightest superpartner (LSP), while a charged slepton is the next-to-lightest superpartner (NLSP). Such models are typically considered to be ruled out. If the gravitino is ignored (as is typical in supersymmetry analyses), the slepton is stable. It then appears as an absolutely stable charged massive particle (CHAMP). There are very strong bounds on CHAMP masses [11, 12, 13, 14]. This constraint removes a large region of mSugra parameter space.

However, as pointed out in [15], CHAMP constraints are relaxed in the presence of the gravitino [16, 17, 18, 19, 20, 21, 22, 23, 24, 25, 26, 27, 28, 29, 30, 31]. The charged slepton will decay to the gravitino in a short time period. The current dark matter will then be entirely composed of gravitinos, and CHAMP searches will not put any constraints on this scenario. Much of the parameter space in the region with slepton NLSPs is consistent with all current limits.

The first comprehensive analysis of the mSugra parameter space with metastable sleptons was performed in a previous paper [32], showing that a large portion of this parameter space can be probed at the first high energy run of the Large Hadron Collider (LHC) with no more than 1-4 $\mathrm{fb}^{-1}$ of data. Some regions of parameter space may even have a "Day 1" discovery of the meta-stable charged sleptons.

In this paper, we shall extend these analyses to consider the possibility of measuring the spin of the NLSP sleptons. We will consider a direct measurement of the spin of the slepton NLSP from Drell-Yan production. The angular and energy distribution of DrellYan produced scalars and fermions are well known, and hence the spin can be measured from the kinematic data. The analysis will be applied to the extended mSugra benchmark models, Model A and Model B, that were defined in [15]. It will be shown that the spin determination is possible for both models with about $40 \mathrm{fb}^{-1}$ of data.

The structure of this paper is the following. A detailed analysis of the mass measurement is performed in Sec. II. Cuts for the spin measurement are developed in Sec. III. The analysis to determine the spin is presented in Sec. IV, along with more general estimates for the prospect of the slepton spin determination as a function of the slepton mass. Sec. $\mathrm{V}$ contains a final discussion of our results. 

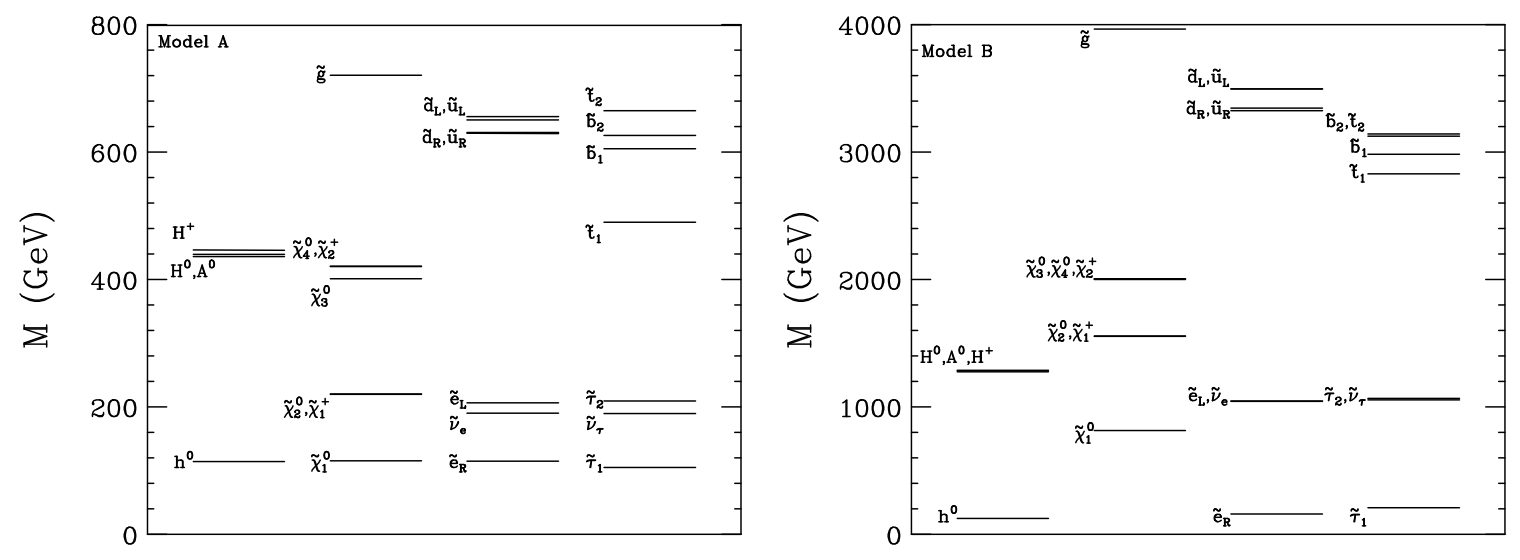

FIG. 1: The SUSY mass spectrum for Model A (Left) and Model B (Right).

\section{MASS MEASUREMENT}

To find the angular distribution in the center of mass frame it is necessary to boost back Drell-Yan produced sleptons from the lab frame. The boost requires a knowledge of the mass of the sleptons. Knowledge of the mass also allows a separation of background muons from the signal [33]. We therefore begin with a discussion of the mass measurement.

The mass spectrum of the two benchmark models can be seen in Fig. 1, where the left panel is the spectrum for Model A, and the right panel is the spectrum for Model B. The discovery potential for these models was studied in [32]. Discovery can be achieved at the LHC with $5.6 \mathrm{pb}^{-1}$ for Model A and $720 \mathrm{pb}^{-1}$ for Model B; both models are well within the reach of the LHC first physics run. The total SUSY cross sections for the two models are $18 \mathrm{pb}$ for Model A and $43 \mathrm{fb}$ for Model B.

Once the meta-stable charged particles have been discovered, a mass measurement can be made with a few low velocity events. The resolution for the momentum and velocity measurements are [33, 34],

$$
\begin{gathered}
\sigma(p)(\mathrm{GeV})=0.000118 p^{2}+0.0002 \sqrt{p^{2}+m_{\tilde{\tau}}^{2}}+0.89 \\
\sigma(\beta)=0.028 \beta^{2} .
\end{gathered}
$$

The low velocity and low momentum events have better detector resolution, thus having a higher resolution on the mass.

A mass measurement to better than $5 \%$ is obtainable with as few as 100 events by taking the ratio of the measured momentum to the measured $\beta \gamma$. The mass measurement accuracy is illustrated in Fig. 2 where the mass of the $105.4 \mathrm{GeV}$ NLSP stau from Model A is measured in the left panel and the 159.5 GeV NLSP selectron from Model B is measured in the right panel. The masses are measured to within a $\mathrm{GeV}$ with 100 events, and the mass resolution for the slower velocity sleptons is a few $\mathrm{GeV}$, matching [34]. The luminosity needed for the mass measurements are $40 \mathrm{pb}^{-1}$ for the stau of Model $\mathrm{A}$ and $15 \mathrm{fb}^{-1}$ for the right handed selectron of Model $\mathrm{B}$, with the use of the following cuts:

a. A slepton candidate: $M_{\text {meas }}=\frac{\beta \gamma_{\text {meas }}}{p_{\text {meas }}}>100 \mathrm{GeV}$

b. Rapidity cuts: $|\eta|<2.4$.

c. Velocity cuts: $0.67<\beta<0.71$

d. Transverse Momentum cut: $p_{T}>20 \mathrm{GeV}$. 

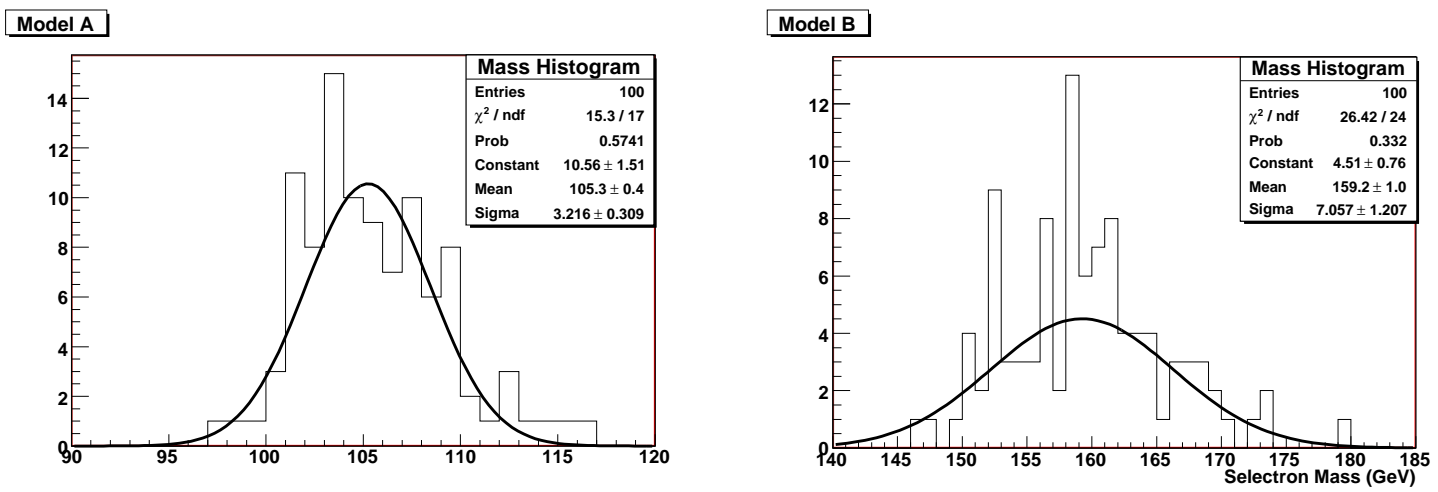

FIG. 2: The histogram of $\frac{p_{\text {meas }}}{\beta \gamma_{\text {meas }}}$ for a $105 \mathrm{GeV}$ stau (left) and a $159 \mathrm{GeV}$ selectron (right).

These cuts do not requires that the sleptons are from Drell-Yan production or that both sleptons are observed in each event. They do require that at least one slepton does trigger the detector. The luminosities quoted above are an overestimate as a result of this requirement.

\section{CUTS}

Drell-Yan events must be isolated from both muons and cascade decays. Muon separation can be obtained in a number of ways. In a previous paper [32], it was shown that the requirement of a time delay can effectively remove muons from our signal. For this paper we will require a time delay greater than 1ns for the observed sleptons. Both particles are also required to have a measured velocity and momentum that are consistent with the measured NLSP slepton mass. These cuts remove both the SM muon and SUSY muon background, while maximizing the acceptance of our signal.

The cascade decays are removed by requiring events to look like Drell-Yan events. Hence the events should have two meta-stable charged particles candidates, without isolated or high energy leptons, without high energy jets, and without missing transverse energy. The following set of cuts are adopted to accomplish this:

a. Two slepton candidates: Two particles with $M_{\text {meas }}$ consistent with $M_{\text {slepton }}$

b. Rapidity cuts: Both candidates with $|\eta|<2.4$

c. Time Delay Cut: Both candidates with $\Delta t>1 \mathrm{~ns}$

d. Transverse Momentum cut: Both candidates with $p_{T}>20 \mathrm{GeV}$

e. No High Energy Jets: E Jet $<20 \mathrm{GeV}$

f. No Missing Energy: Missing Transverse Energy $<15 \mathrm{GeV}$

g. No High Energy or Isolated Leptons: $p_{\text {lepton }}<6 \mathrm{GeV}$ if not isolated

These cuts succeed at separating the sleptons from cascade decays from the Drell-Yan produced particles so long as the particle spectrum is well spaced, leading to large jet energies in cascade decays. This type of spectrum is generic in mSugra space.

It should be noted that these cuts are very severe in general for the extended mSugra space. The cuts are meant to separate out the sleptons produced from decays without knowing the mass spectrum, and can be excessive for mass spectra like Model B where less than 1 out of every 10000 slepton events that passes the cuts is not a Drell-Yan event. Less stringent cuts could be used with an increase in the signal if the mass spectrum is taken into account. On the other hand, if the mass spectrum is more compressed than that of Model 

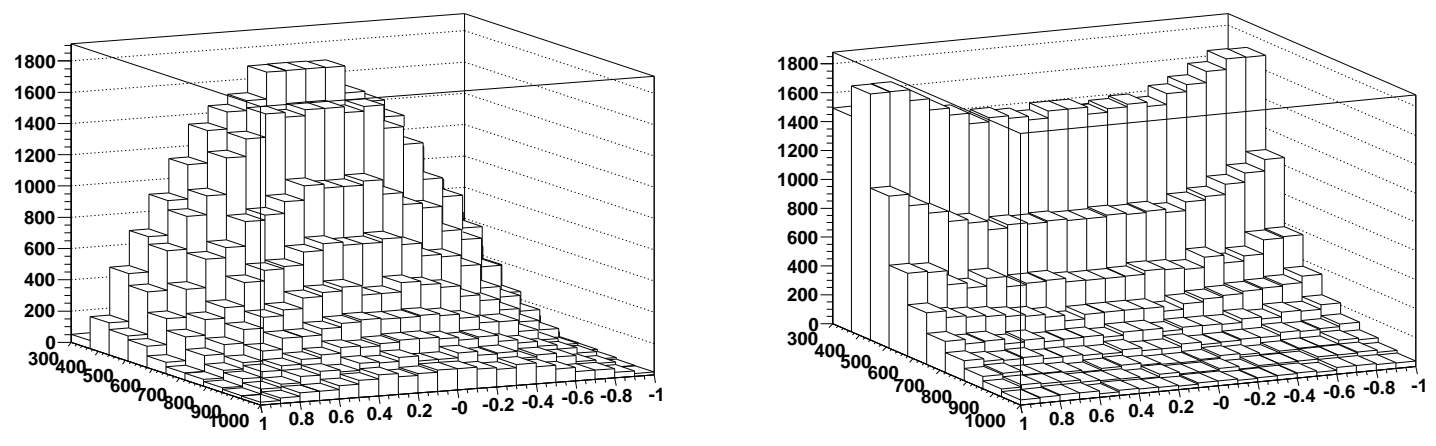

FIG. 3: A $20 \times 20$ two dimensional histogram of the center of mass kinematic variables for a 159 $\mathrm{GeV}$ scalar (left) and fermion (right). The $\mathrm{x}$-axis is $\cos \theta^{*}$ with a range from -1 to 1 . The $\mathrm{y}$-axis is $\sqrt{s}=2 E^{*}$ with a range from $300 \mathrm{GeV}$ to $1000 \mathrm{GeV}$.

A, the success of the cuts decreases. These types of mass spectra are not characteristic in the extended mSugra space, though they can be found in other models. Specialized cuts for specific mass spectra would improve the results. This is not done here since the goal of this paper is to determine a general and conservative prospect for spin determination at the LHC.

After applying these cuts, the effective Drell-Yan cross sections for the NLSP sleptons are $4.72 \mathrm{fb}$ for Model A and $1.05 \mathrm{fb}$ for Model B. The cross sections for the sleptons from cascade decays are $0.187 \mathrm{fb}$ for Model A and less than $0.001 \mathrm{fb}$ for Model B. The dual requirements of both slepton candidates having a mass consistent with the measured slepton mass and a time delay greater than 1 ns reduces the SM and BSM muon background to negligible values.

We can therefore ignore the backgrounds in the further analysis, since they are very small. We will assume that all events that pass the cuts above are Drell Yan events, and we now proceed to an analysis of the spin measurement.

We note that the above cuts, which are necessary to remove these NLSP sleptons not produced by Drell-Yan processes, alter the actual distributions from naive theoretical calculations. As a result, the experimental distribution must be compared to a theoretical distribution with the same experimental cuts applied.

\section{SPIN DETERMINATION}

Scalars produced in Drell-Yan processes have a $\sin ^{2} \theta^{*}$ angular distribution in the center of mass frame independent of the center of mass energy while Drell-Yan produced fermions have a $1+\left(\frac{E^{* 2}-m^{2}}{E^{* 2}+m^{2}}\right) \cos ^{2} \theta^{*}$ angular distribution in the center of mass frame. These distributions are shown in Fig. 3 for a $159.5 \mathrm{GeV}$ scalar (left) and fermion (right). We wish to determine how much luminosity is necessary to determine the NLSP slepton's spin, and in particular, to establish that the angular distribution is consistent with a scalar rather than a fermion distribution.

The luminosity is effectively set by two numbers: The effective Drell-Yan cross section after the cuts, and the number of Drell-Yan events needed to distinguish between a spin-0 and spin- $1 / 2$ particle. The average number of events needed for a spin determination is 

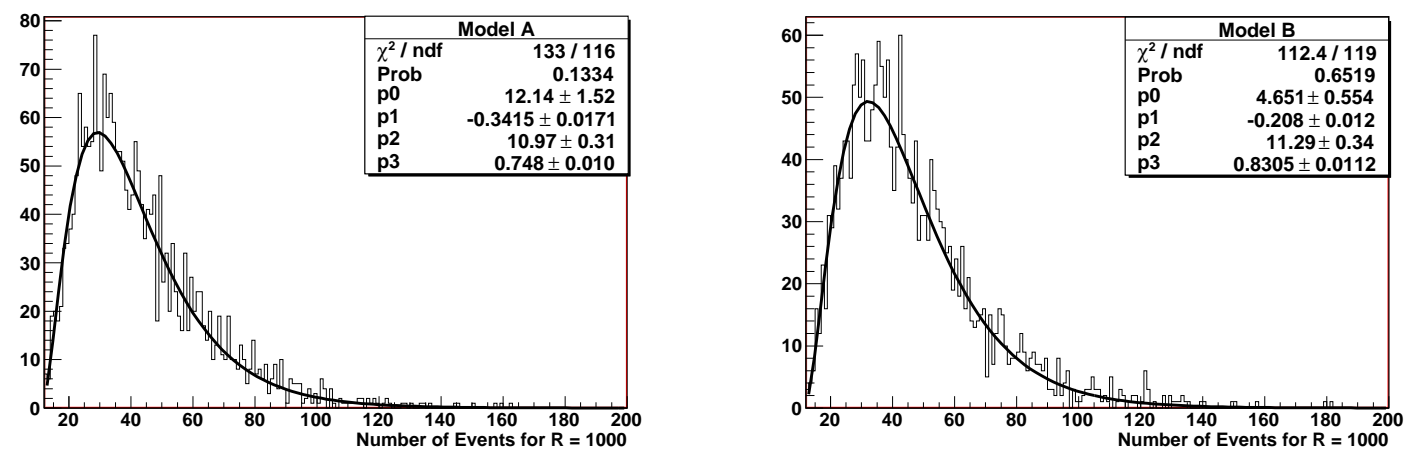

FIG. 4: The histograms of the number of events needed for $\mathrm{R}=1000$. The results are fitted well by Eq. (6) with the fitted parameters listed in the upper right hand corner of each panel.

given by a log ratio test for the two distributions [35]

$$
\bar{N}=\frac{\log (R)}{\int \log \left(\frac{f_{s}(x)}{f_{f}(x)}\right) f_{s}(x) d x} .
$$

$R$ is the desired probability of having one distribution over the other distribution for a given set of observables. We will require $R=10^{3}$, which would imply that an experimental distribution is $10^{3}$ times more likely to be a scalar distribution than a fermion distribution. The integral is over the entire observable space, parameterized by $E^{*}$ and $\cos \theta^{*}$ in the present case, and the two functions, $f_{s}(x)$ and $f_{f}(x)$, are the scalar and fermion probability distribution functions after the imposition of the cuts.

The probability distribution functions are generated using PYTHIA 6.406. Drell-Yan events were generated for a scalar and a fermion with the measured mass of the model (including the previous uncertainties). If the event passed the cuts, it was then included into a $20 \times 20$ two dimensional $\left(E^{*}, \cos \theta^{*}\right)$ grid until $10^{5}$ such events were generated. The large number of events is necessary to lower the relative fluctuation per grid bin resulting in a more accurate probability distribution function. The functions $f_{s}$ and $f_{f}$ are obtained by normalizing the events in each grid bin.

The average number of events needed for a spin determination can then be calculated by use of Eq. (33). For the two bench mark models, the numbers are found to be

$$
\begin{gathered}
\bar{N}_{A}=\frac{\log \left(10^{3}\right)}{\sum_{i} \log \left(\frac{f_{s}^{A}\left(x_{i}\right)}{f_{f}^{A}\left(x_{i}\right)}\right) f_{s}^{A}\left(x_{i}\right)} \approx 41 \\
\bar{N}_{B}=\frac{\log \left(10^{3}\right)}{\sum_{i} \log \left(\frac{f_{s}^{B}\left(x_{i}\right)}{f_{f}^{B}\left(x_{i}\right)}\right) f_{s}^{B}\left(x_{i}\right)} \approx 44,
\end{gathered}
$$

and translate to average luminosities of $L_{A}=8.7 \mathrm{fb}^{-1}$ for Model $\mathrm{A}$ and $L_{B}=42 \mathrm{fb}^{-1}$ for Model B. On average the spin of the slepton NLSP of Model A can be measured by the end of the second LHC physics run.

In general the number of events necessary to distinguish between the scalars and fermions will fluctuate widely around $\bar{N}$; that is, over several different runs, there is a large variation 


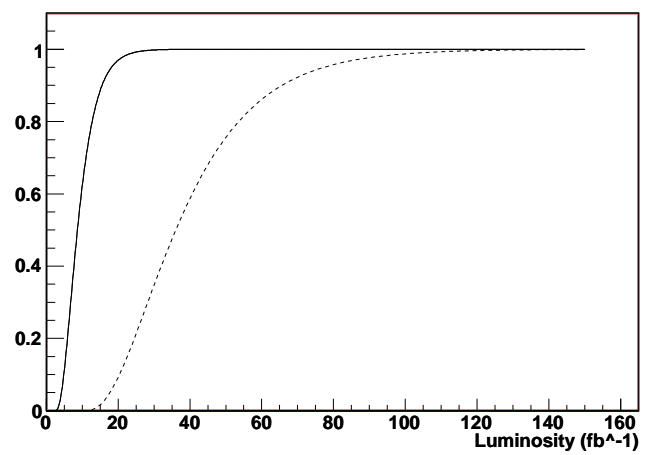

FIG. 5: The probability that the spin will be determined at the LHC as a function of luminosity. The solid line is for the NLSP stau of Model A, and the dashed line is for the NLSP right handed selectron of Model B.

in the number of events required to get $R=10^{3}$. For any given luminosity, we should therefore provide a probability of distinguishing between fermions and scalars.

The distributions of the number of events needed to determine the spin are shown in Fig. 4 for Model A (left) and Model B (right). The distributions are constructed by generating Drell-Yan events for each model until $R \geq 10^{3}$. The number of events needed to reach this value of $R$ is then binned into a histogram until 2000 counts have been made.

The distributions have a tail towards higher number of events and are not fitted well by any common probability distribution function. They were fitted best with a fitting function of the form

$$
L(N)=p_{0}\left(N-p_{2}\right) e^{p_{1} N^{p_{3}}}
$$

with $p_{0}$ setting the overall scale of the function, $p_{1}$ being the negative coefficient in the exponential, $p_{2}$ setting the value which the function starts, and $p_{3}$ determining with what power the exponential tails falls with. The best fit values for these parameters can be found in Fig. 4 .

The total probability of getting a distribution capable of distinguishing between a fermion and a scalar for a given number of events is then given by the integration of the normalized probability distribution function in Eq. (6) , from the cutoff to the given number of events.

$$
P(N)=C \int_{p_{2}}^{N} L\left(N^{\prime}\right) d N^{\prime}
$$

where $C$ is a normalization factor.

The probability $P(N)$ as a function of luminosity is plotted in Fig. 5. It is constructed by using the effective Drell-Yan cross section of $4.72 \mathrm{fb}$ for Model A and $1.05 \mathrm{fb}$ for Model B. By the end of the second $30 \mathrm{fb}^{-1}$ physics run the probability of getting a sample of Drell-Yan events from which the spin can be determined are $99.8 \%$ for Model A and $34.6 \%$ for Model B. The results differ for the two models because of the difference in the Drell-Yan cross sections, an overall factor since $L(N)$ is a function of the number of events.

In general, the Drell-Yan cross section at the LHC is a function of mass and of the amount of left and right handedness in the mass eigenstate. Using the results from the fit combined with these Drell-Yan cross sections, an estimate is made of the probability of producing a spin measurement by the end of the second LHC physics run as a function of mass and 

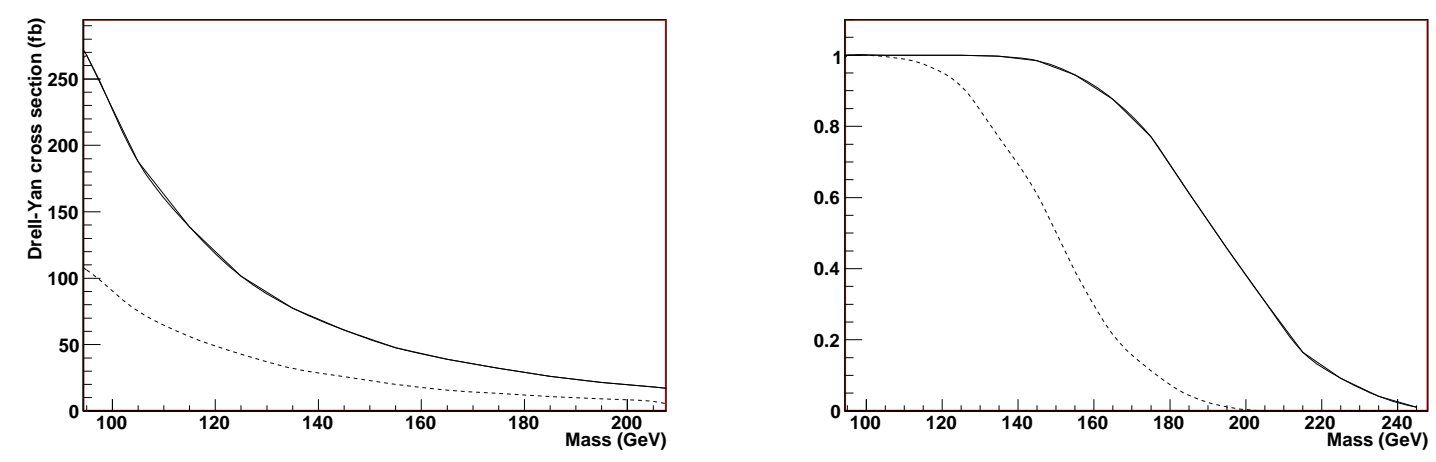

FIG. 6: The left panel is the slepton Drell-Yan cross section as a function of mass. The right panel is the probability that the spin will be determined by the end of the second $30 \mathrm{fb}^{-1}$ physics run as a function of slepton mass. In both panels the solid line is for a left handed slepton, and the dashed line represents a right handed slepton. Results for mixed states lie between the two lines.

handedness. This is shown in Fig. 6. A spin determination is 95\% probable for a left handed NLSP slepton with mass below 145 - $155 \mathrm{GeV}$. Similarly, a right handed NLSP slepton spin determination is probable for a mass below 115-125 GeV.

\section{CONCLUSION}

The first physics run of the LHC is expected to have a luminosity of $1-4 \mathrm{fb}^{-1}$. For both benchmark models, the NLSP slepton will be discovered in this run.

The second physics run will aim at acquiring $30 \mathrm{fb}^{-1}$ of luminosity. In this run, we have shown that there is a good chance that the spin of the slepton can be measured. For Model A there is a $99.8 \%$ chance of determining the spin of the $105 \mathrm{GeV}$ stau, and for Model B there is a $34.6 \%$ chance of determining the spin of the $159 \mathrm{GeV}$ right handed selectron. On average these spin determinations require $8.7 \mathrm{fb}^{-1}$ and $42 \mathrm{fb}^{-1}$ respectively for these two models.

We have also estimated the chances of determining the spin of the NLSP sleptons in a generic scenario with metastable sleptons. Slepton masses less than $120 \mathrm{GeV}$ will likely be verified as scalars by the end of the second physics run, regardless of the handedness. Left handed sleptons can be shown to be scalar if the mass is less than $150 \mathrm{GeV}$. Since most of the mSugra parameter space with a NLSP slepton is largely right handed, it is not likely that the spin of the slepton will be determined in the second physics run at the LHC if its mass is greater than $200 \mathrm{GeV}$.

Similar results can be derived for $100 \mathrm{fb}^{-1}$ and $300 \mathrm{fb}^{-1}$. With $100 \mathrm{fb}^{-1}$, it is possible to determine the slepton spin if the mass is below $290 \mathrm{GeV}$, and with $300 \mathrm{fb}^{-1}$ the reach will extend to $380 \mathrm{GeV}$.

To conclude, we have shown that it is possible to measure the spin of metastable charged particles (e.g. metastable sleptons) after two years of LHC running. It would be also be interesting to extend this analysis to see if the spins of the other superpartners can be measured, using information from cascade decays (for example, see [5]). Such an analysis could confirm the nature of the new physics to be supersymmetry while excluding extra dimension models. 


\section{Acknowledgments}

The work of AR is supported in part by NSF Grants No. PHY-0354993 and PHY0653656. BTS is supported in part by NSF CAREER Grant No. PHY-0239817.

[1] H. C. Cheng, K. T. Matchev and M. Schmaltz, Phys. Rev. D 66, 056006 (2002) arXiv:hep-ph/0205314.

[2] A. J. Barr, Phys. Lett. B 596, 205 (2004) arXiv:hep-ph/0405052.

[3] J. M. Smillie and B. R. Webber, JHEP 0510, 069 (2005) arXiv:hep-ph/0507170.

[4] A. Datta, K. Kong and K. T. Matchev, Phys. Rev. D 72, 096006 (2005) [Erratum-ibid. D 72, 119901 (2005)] arXiv:hep-ph/0509246.

[5] A. J. Barr, JHEP 0602, 042 (2006) [arXiv:hep-ph/0511115].

[6] A. Alves, O. Eboli and T. Plehn, Phys. Rev. D 74, 095010 (2006) arXiv:hep-ph/0605067.

[7] C. Athanasiou, C. G. Lester, J. M. Smillie and B. R. Webber, JHEP 0608, 055 (2006) arXiv:hep-ph/0605286.

[8] L. T. Wang and I. Yavin, JHEP 0704, 032 (2007) arXiv:hep-ph/0605296].

[9] A. Alves and O. Eboli, arXiv:0704.0254 [hep-ph].

[10] A. H. Chamseddine, R. Arnowitt and P. Nath, Phys. Rev. Lett. 49, 970 (1982); R. Barbieri, S. Ferrara and C. A. Savoy, Phys. Lett. B 119, 343 (1982); N. Ohta, Prog. Theor. Phys. 70, 542 (1983); L. J. Hall, J. D. Lykken and S. Weinberg, Phys. Rev. D 27, 2359 (1983); L. Alvarez-Gaume, J. Polchinski and M. B. Wise, Nucl. Phys. B 221, 495 (1983).

[11] P. F. Smith and J. R. J. Bennett, Nucl. Phys. B 149, 525 (1979); P. F. Smith, J. R. J. Bennett, G. J. Homer, J. D. Lewin, H. E. Walford and W. A. Smith, Nucl. Phys. B 206, 333 (1982); T. K. Hemmick et al., Phys. Rev. D 41, 2074 (1990); P. Verkerk, G. Grynberg, B. Pichard, M. Spiro, S. Zylberajch, M. E. Goldberg and P. Fayet, Phys. Rev. Lett. 68, 1116 (1992); T. Yamagata, Y. Takamori and H. Utsunomiya, Phys. Rev. D 47, 1231 (1993).

[12] S. Dimopoulos, D. Eichler, R. Esmailzadeh and G. D. Starkman, Phys. Rev. D 41, 2388 (1990); M. Byrne, C. F. Kolda and P. Regan, Phys. Rev. D 66, 075007 (2002) hep-ph/0202252.

[13] M. Pospelov, arXiv:hep-ph/0605215.

[14] R. H. Cyburt, J. Ellis, B. D. Fields, K. A. Olive and V. C. Spanos, arXiv:astro-ph/0608562.

[15] J. L. Feng, A. Rajaraman and B. T. Smith, Phys. Rev. D 74, 015013 (2006) arXiv:hep-ph/0512172.

[16] J. L. Feng, A. Rajaraman and F. Takayama, Phys. Rev. Lett. 91, 011302 (2003) hep-ph/0302215; Phys. Rev. D 68, 063504 (2003) hep-ph/0306024.

[17] J. R. Ellis, K. A. Olive, Y. Santoso and V. C. Spanos, Phys. Lett. B 588, 7 (2004) hep-ph/0312262.

[18] J. L. Feng, S. f. Su and F. Takayama, Phys. Rev. D 70, 063514 (2004) hep-ph/0404198; Phys. Rev. D 70, 075019 (2004) hep-ph/0404231].

[19] F. Wang and J. M. Yang, Eur. Phys. J. C 38, 129 (2004) hep-ph/0405186.

[20] J. R. Ellis, K. A. Olive, Y. Santoso and V. C. Spanos, Phys. Lett. B 603, 51 (2004) hep-ph/0408118.

[21] L. Roszkowski, R. Ruiz de Austri and K. Y. Choi, JHEP 0508, 080 (2005) hep-ph/0408227.

[22] A. Brandenburg, L. Covi, K. Hamaguchi, L. Roszkowski and F. D. Steffen, Phys. Lett. B 617, 99 (2005) hep-ph/0501287. 
[23] D. G. Cerdeno, K. Y. Choi, K. Jedamzik, L. Roszkowski and R. Ruiz de Austri, hep-ph/0509275.

[24] M. Kaplinghat, Phys. Rev. D 72, 063510 (2005) astro-ph/0507300.

[25] J. A. R. Cembranos, J. L. Feng, A. Rajaraman and F. Takayama, Phys. Rev. Lett. 95, 181301 (2005) hep-ph/0507150].

[26] K. Jedamzik, M. Lemoine and G. Moultaka, astro-ph/0508141.

[27] K. Sigurdson and M. Kamionkowski, Phys. Rev. Lett. 92, 171302 (2004) [astro-ph/0311486].

[28] S. Profumo, K. Sigurdson, P. Ullio and M. Kamionkowski, Phys. Rev. D 71, 023518 (2005) astro-ph/0410714.

[29] F. D. Steffen, JCAP 0609, 001 (2006) arXiv:hep-ph/0605306.

[30] F. D. Steffen, AIP Conf. Proc. 903, 595 (2007) arXiv:hep-ph/0611027.

[31] J. Pradler and F. D. Steffen, Phys. Rev. D 75, 023509 (2007) arXiv:hep-ph/0608344.

[32] A. Rajaraman and B. T. Smith, arXiv:hep-ph/0612235.

[33] J. R. Ellis, A. R. Raklev and O. K. Oye, JHEP 0610, 061 (2006) arXiv:hep-ph/0607261.

[34] G. Polesello, A. Rimoldi, "Reconstruction of quasi-stable charged sleptons in the ATLAS Muon Spectrometer", ATL-MUON-99-006.

[35] J. Orear, Notes on Statistics for Physicists, UCRL-8417, http://nedwww.ipac.caltech.edu/level5/Sept01/Orear/frames.html 